\title{
TRAUMA E DOAÇÃO DE ÓRGÃOS E TECIDOS PARA TRANSPLANTE
}

\author{
Trauma and Tissue and Organ Donation for Transplant \\ Edvaldo Leal de Moraes, Leonardo Borges de Barros e Silva, Milton Glezer, \\ Nair Cordeiro dos Santos da Paixão, Tatiana Cristine de Moraes
}

\begin{abstract}
RESUMO
Atualmente, trauma representa uma das grandes causas de morte. Algumas vítimas de trauma podem evoluir para morte encefálica, podendo ser viabilizadas como doadoras de órgãos e tecidos. Objetivos: Conhecer os diferentes tipos de trauma que resultaram em morte encefálica; caracterizar potenciais doadores segundo a faixa etária, sexo e raça; quantificar os potenciais doadores que efetivaram ou não a doação de órgãos e tecidos, especificando os motivos da não-doação; conhecer quais os órgãos e tecidos utilizados para transplante. Métodos: Estudo quantitativo, descritivo e retrospectivo, utilizando dados coletados dos prontuários da Organização de Procura de Órgãos do Hospital das Clínicas da Faculdade de Medicina da Universidade de São Paulo. Resultados: Foram analisados os dados de 198 prontuários de potenciais doadores, durante o período de janeiro de 2004 a dezembro de 2005. Os dados mostraram que dos potenciais doadores vítimas de trauma, $78,79 \%$ eram do sexo masculino, $67,17 \%$ eram da raça branca e a faixa etária predominante foi de 0 a 25 anos com $47,48 \%$. Dentre os diferentes tipos de trauma, o acidente de trânsito representou 44,45\%. Dos potenciais doadores, 72,73\% não efetivaram a doação e, dos motivos de não-doação, a recusa familiar representou 45,83\% e parada cardíaca, $41,67 \%$. Dos doadores, os órgãos mais utilizados foram os rins com 98,14\%. Conclusão: Os resultados mostraram que a não efetivação da doação se deu principalmente por recusa familiar e por parada cardíaca. Fica evidente que o tema merece atenção da sociedade, do governo e dos profissionais da saúde.
\end{abstract}

Descritores: Transplante, Traumatismos, Morte Encefálica e Enfermagem.

\section{Instituição:}

Organização de Procura de Órgãos do Hospital das Clínicas da Faculdade de Medicina da Universidade de São Paulo - São Paulo, SP, Brasil

\section{Correspondência:}

Edvaldo Leal de Moraes

Av. Dr. Enéas de Carvalho Aguiar, 255 - sala 5017-A - Cerqueira César CEP 05403-000 - São Paulo - SP

Tel./Fax: 5511 3069-8004

E-mail: edvaldoleal@uol.com.br

Recebido em: 08.08.2006

\section{INTRODUÇÃO}

Trauma tem sido considerado um sério problema social e comunitário, e hoje um dos mais significativos problemas de toda a área da saúde, sendo a principal causa de morte na faixa etária até os 45 anos $^{1}$.

$\mathrm{O}$ trauma pode ser rotulado como a doença negligenciada do mundo moderno, tanto que os investimentos feitos visando seu controle, prevenção e tratamento são inversamente proporcionais à rápida progressão da violência e dos mais diferentes tipos de traumatismo ${ }^{1}$. Traumatismo é o termo clínico que traduz o conjunto de alterações causadas por um agente físico em uma pessoa ${ }^{2}$.

Hoje, graças à implantação de sistemas de atendimento mais atuantes e mais resolutivos, e também em decorrência da crescente implantação de cursos de capacitação e atualização, como é o caso do Suporte Avançado de Vida em Trauma (ATLS), do Suporte Pré-hospitalar de Vida em Trauma (PHTLS) e do Suporte de Vida em Trauma para Enfermeiros (TLSN), o atendimento ao trauma tem sido mais adequado e eficiente. Obviamente, o treinamento de profissionais de saúde é apenas uma etapa em um longo e complexo processo de aprimoramento assistencial, que está longe de ser totalmente equacionado ${ }^{3}$.

O trauma representa cerca de $10 \%$ dos óbitos no mundo todo. Em termos "etiológicos", os traumatismos (causas externas) podem ser reunidos em dois grandes grupos: os denominados "nãointencionais" (atropelamento, colisões, quedas, afogamentos, etc.) e os "intencionais" (suicídios, homicídios, violências e guerras). No mundo, entre as diferentes causas externas, os acidentes por veículos 
automotores, colisões e atropelamentos constituem a primeira causa de morte, seguidos por suicídios e homicídios ${ }^{3}$.

No Brasil, cerca de 130 mil pessoas são vítimas de trauma a cada ano. Segundo dados do Ministério da Saúde (2005), desse total, 40\% são devido a acidentes de trânsito, $40 \%$ por ferimentos penetrantes (tiro e faca), e os demais por quedas, afogamentos e outras causas. Esta é a principal causa de morte na população masculina até 45 anos. Analisando a população em geral, o trauma vem a ser a segunda causa, atrás apenas das doenças cardiovasculares. Por isso, ele é considerado pelos especialistas como a doença do século. Segundo o Professor Doutor Dario Birolini, da disciplina de Cirurgia Geral da Faculdade de Medicina da Universidade de São Paulo (FMUSP), o trauma é uma doença peculiar, pois ocorre da forma mais imprevisível possível. Agride a vítima sem proteção, como "é o caso do tiro; ninguém sabe onde a bala vai pegar e qual o estrago que vai fazer"

É nesse contexto de violência que muitos indivíduos vitimados pelo trauma evoluem para danos neurológicos graves e irreversíveis, levando à morte encefálica $(\mathrm{ME})$. A morte encefálica é caracterizada pela parada completa e irreversível de todas as funções neurológicas intracranianas, considerando tanto os hemisférios cerebrais como o tronco encefálico.

Estudo realizado no Departamento de Neurocirurgia da Universidade Lódz (Polônia) mostrou que dos pacientes vítimas de trauma com índice 3 de Glasgow na admissão, 89\% morreram, 7\% sobreviveram em coma vegetativo persistente e $4 \%$ sobreviveram e receberam alta hospitalar. Concluíram que entre os pacientes com índice 3 de Glasgow, somente aqueles sem maiores anormalidades na tomografia craniana ou aqueles com hematoma epidural têm chance de sobreviver. Os pacientes portadores de hematoma intracraniano tinham péssimo prognóstico (não existindo, praticamente, chance de sobrevivência). Nesses casos, os pacientes devem ser considerados como possíveis doadores de órgãos, mas somente após a parada da circulação cerebral. E, quando a morte encefálica for comprovada de acordo com os padrões internacionalmente aceitos, eles podem ser tratados como potenciais doadores ${ }^{5}$.

A escala de coma de Glasgow foi introduzida como modelo para monitoramento do nível de consciência após trauma de crânio. Embora o objetivo inicial fosse criar um método para monitoramento do nível de consciência, sua utilização provouse eficaz também como índice de gravidade de lesão cerebral, relacionando-se com o prognóstico ${ }^{3}$. A escala de coma de Glasgow baseia-se na observação de três tipos de resposta: abertura ocular, melhor resposta ao comando verbal e melhor resposta motora. O valor mínimo é de 3 e o máximo é de 15. Quanto maior o valor, menor será o nível de gravidade, e melhor o prognóstico. Por definição, um paciente em coma não apresenta abertura ocular, não tem capacidade para obedecer a comandos e não verbaliza ${ }^{3}$. A identificação dos pacientes com índice 3 de Glasgow no Brasil possibilita a realização de exame clínico para investigação de morte encefálica, desde que sejam respeitadas as determinações da Resolução do Conselho Federal de Medicina (CFM) no 1480/97, que estabelece os critérios para o diagnóstico de ME atualmente aceitos. Estes critérios incluem: a) causa do coma; b) causas do coma que devem ser excluídas durante o exame: hipotermia e uso de drogas depressoras do sistema nervoso central (SNC); c) elementos do exame neurológico: coma aperceptivo, pupilas fixas e arreativas, ausência de reflexo córneo-palpebral, ausência de reflexo oculocefálico, ausência de reflexo de tosse e apnéia. Além das provas protocolares e padronizadas em todo território nacional, é obrigatória a realização de um exame complementar, que vai caracterizar de forma inequívoca: 1) ausência de fluxo vascular cerebral; 2) ausência de atividade eletrofisiológica; ou 3) ausência de atividade metabólica ${ }^{6}$. Uma vez identificado o indivíduo com índice 3 de Glasgow que preencha os critérios clínicos de $\mathrm{ME}$, é obrigatório por lei que todos os estabelecimentos de saúde notifiquem as Centrais de Notificação, Captação e Distribuição de Órgãos da Unidade Federada do ocorrido, a suspeita de morte encefálica, feito em pacientes por eles atendidos?

Nesse momento as Organizações de Procura de Órgãos (OPOs) assumem um papel fundamental, que consiste na viabilização do possível doador para o Sistema Estadual de Transplante. Vale lembrar que há dez OPOs no estado de São Paulo, sendo quatro na cidade de São Paulo, situadas em hospitais escolas (OPO-HCFMUSP, OPO-Santa Casa, OPO-Dante Pazzanese e OPO-Escola Paulista de Medicina), e no interior do Estado há mais seis OPOs (OPOSorocaba, OPO-Marília, OPO-Ribeirão Preto, OPO-Botucatu, OPO-Campinas e OPO-São José do Rio Preto). Os profissionais que compõem essas organizações são em sua maioria médicos e enfermeiros que têm um importante papel no complexo processo de doação-transplante. O processo de obtenção de órgãos para transplante inicia-se com os médicos intensivistas ou assistentes que identificam os potenciais doadores, fazem a declaração de morte encefálica, notificam a família e fazem a comunicação à coordenação de transplante. Continua com a entrevista com a família, em que se solicita o consentimento para remoção dos órgãos e tecidos, e com a avaliação das condições clínicas do potencial doador e da viabilidade dos órgãos a serem extraídos, e termina após a remoção e distribuição dos órgãos e tecidos, com a análise dos resultados ${ }^{8}$. Ressalta-se que a família do possível doador assume papel importante dentro desse processo, pois, conforme a Lei no 10.211 de março de 2001, a retirada de tecidos, órgãos e partes do corpo de pessoas falecidas para transplante ou outra finalidade terapêutica, dependerá da autorização do cônjuge ou parente maior de idade, obedecida a linha sucessória, reta ou colateral, até o segundo grau. Inclusive firmada em documento subscrito por duas testemunhas 9 .

Foi a partir desse complexo contexto que envolve o paciente, vítima de trauma e o processo de doação de órgãos e tecidos para transplante, que se decidiu realizar este estudo, com os seguintes objetivos: conhecer os diferentes tipos de trauma que resultaram em morte encefálica; caracterizar a população vítima de trauma com suspeita de morte encefálica segundo a faixa etária, sexo e raça; quantificar os potenciais doadores vítimas de trauma que efetivamente foram ou não doadores de órgãos e tecidos, especificando os motivos da não-doação; e conhecer quais os órgãos e tecidos utilizados para transplante de doadores vítimas de trauma.

\section{MÉTODOS}

Trata-se de um estudo de caráter quantitativo, descritivo, exploratório e retrospectivo ${ }^{10,11,12}$. Os dados obtidos permitiram a caracterização dos pacientes vítimas de trauma que passaram pelo processo de doação-transplante. A pesquisa foi realizada na Organização de Procura de Órgãos do Hospital das Clínicas da Faculdade de Medicina da Universidade de São Paulo (OPOHCFMUSP). A amostra estudada foi constituída dos prontuários de potenciais doadores de órgãos e tecidos, sem distinção de sexo ou faixa etária, vítimas de traumas durante o período de janeiro de 2004 a dezembro de 2005. Utilizou-se uma planilha contendo os 
seguintes dados dos potenciais doadores: sexo, raça, faixa etária, mecanismo do trauma, se foi doador ou não-doador, motivo da não-doação e órgãos utilizados para transplante. Após essa fase, os dados coletados foram analisados e transformados em gráficos disponíveis em programa do Windows.

A Comissão de Ética para Análise de Projetos de Pesquisa - CAPPesq da Diretoria Clínica do Hospital das Clínicas da Faculdade de Medicina da Universidade de São Paulo aprovou o presente estudo.

\section{RESULTADOS}

Na OPO-HCFMUSP, durante o período de janeiro de 2004 a dezembro de 2005 , a notificação de potenciais doadores vítima de trauma representou $37,5 \%$ do total de notificações. Abaixo foi discriminado através de gráficos o perfil desses potenciais doadores.

No gráfico 1 observa-se a distribuição dos potenciais doadores de órgãos e tecidos segundo o mecanismo de trauma. Dos 198 potenciais

Gráfico 1: Distribuição dos potenciais doadores de órgãos e tecidos vítimas de trauma, segundo o mecanismo de trauma, OPO-HCFMUSP, 2004-2005.

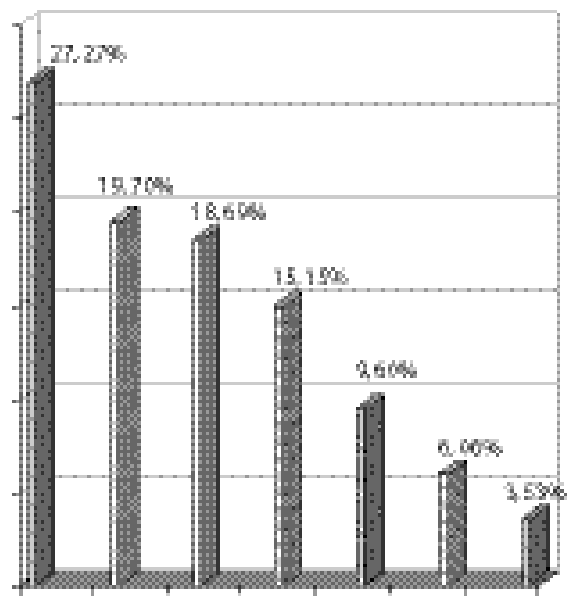

m TCE por queds

DTCE pos itropelamemo

Q Fü sm cránio

D TCE po sciderte moto

DTCE pos niderse aso

DTCE pos agresssio

\section{potros}

doadores avaliados pela OPO-HCFMUSP no período de janeiro de 2004 a dezembro de 2005, 54 (27,27\%) foram em conseqüência de TCE por queda, 39 (19,70\%) TCE por atropelamento, $37(18,69 \%)$ ferimento por arma de fogo (FAF) em crânio, 30 (15,15\%) TCE por acidente de moto, $19(9,60 \%)$ TCE por acidente de automóvel, e 12 $(6,06 \%)$ TCE por agressão física. Outras causas como afogamento, TCE por acidente de bicicleta, FAF cervical e choque elétrico somaram sete casos $(3,53 \%)$. Vale ressaltar que acidentes de trânsito representados por atropelamento, acidente de automóvel e moto representaram $44,45 \%$ de todas as causas de morte encefálica em potenciais doadores vítimas de trauma.

No gráfico 2 observa-se que 94 casos (47,48\%) estavam na faixa etária de 0 a 25 anos; 66 (33,33\%) na faixa etária de 26 a 45 anos; 34 (17,17\%) de 46 a 65 anos; e apenas quatro $(2,02 \%)$ com mais de 65 anos.

Dos 198 potenciais doadores, como apresentado no gráfico 3, $144(72,73 \%)$ não foram doadores efetivos, sendo que apenas 54 $(27,27 \%)$ foram encaminhados para doação.

No gráfico 4 percebe-se que, dos motivos para não-doação, a recusa familiar representou $45,83 \%$ (66) e a parada cardio-respiratória
$41,67 \%$ (60). As outras causas como instabilidade hemodinâmica, fora do protocolo de morte encefálica, sorologia positiva, septicemia e sem responsável legal representaram $12,50 \%$ (18).

Gráfico 2: Distribuição dos potenciais doadores de órgãos e tecidos vítimas de trauma, segundo a faixa etária, OPO-HCFMUSP, 2004-2005.

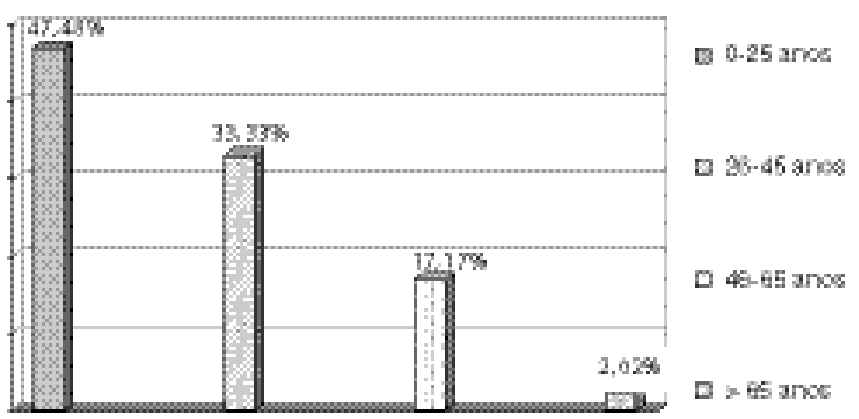

Gráfico 3: Distribuição dos potenciais doadores de órgãos e tecidos vítimas de trauma, segundo a efetivação em doadores e não-doadores, OPO-HCFMUSP, 2004-2005

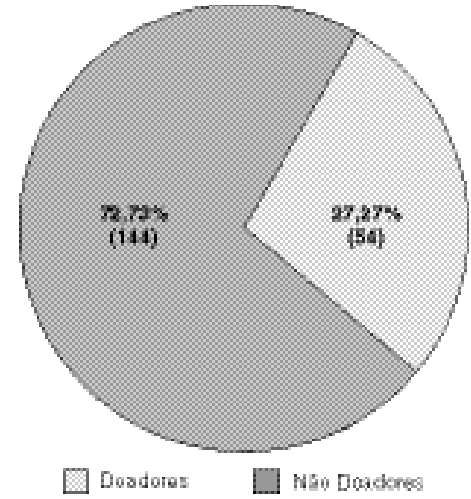

Gráfico 4: Distribuição dos potenciais doadores de órgãos e tecidos vítimas de trauma, segundo os motivos da não doação, OPO-HCFMUSP, 2004-2005.

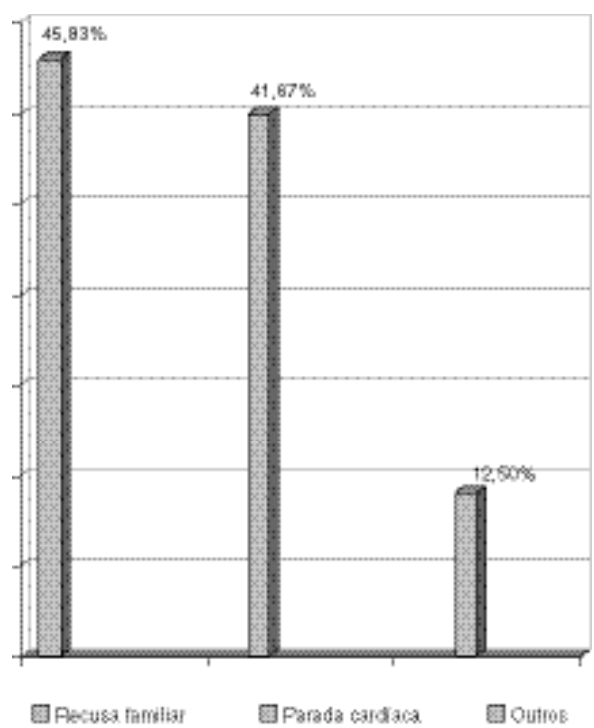




\section{DISCUSSÃO}

As mortes violentas estão incluídas na 9ํㅡㄹ Classificação Internacional de Doenças (CID-9) da Organização Mundial de Saúde (OMS) sob a denominação de "Causas externas de lesões e envenenamento". A mortalidade por causas externas no Brasil é semelhante às do México, da Nicarágua, do Panamá e da Venezuela (ao redor de $15 \%$ ), apenas superada pela Colômbia ${ }^{13}$. O trauma produz cerca de 150 mil mortes por ano nos Estados Unidos ${ }^{14}$. No Brasil, são 130 mil mortes por ano, representando a segunda causa de morte, atrás apenas das doenças cardiovasculares ${ }^{4}$. Dos vários tipos de trauma, o traumatismo craniencefálico (TCE) pode produzir lesões intracranianas letais, levando o indivíduo à morte encefálica. $\mathrm{O}$ levantamento realizado mostrou que as vítimas de trauma por acidente de trânsito representaram $44,45 \%$ de todas as causas de morte encefálica, confirmando os dados da literatura, que mostram que os acidentes de trânsito representam cerca de $40 \%$ das mortes por trauma ${ }^{4}$. De acordo com a Companhia de Engenharia de Tráfego CET, na capital paulista quatro pessoas morrem todos os dias vítimas de acidente de trânsito. A cada quatro mortos, três são homens, e a maioria jovem de 18 a 29 anos, respaldando nossos achados ${ }^{15}$.

$\mathrm{O}$ trauma permanece como a principal causa de morte nas primeiras quatro décadas de vida (idades de um a 44 anos) ${ }^{14}$. O mesmo foi encontrado nesta pesquisa, onde $47,48 \%$ das vítimas estavam na faixa etária de 0 a 25 anos. Estudo realizado sobre mortalidade no município de São Paulo mostrou que a idade que concentrou a maior freqüência de mortes por trauma foi de 21 anos, sendo que o crescimento inicia-se na faixa dos 15 aos 19 anos, decrescendo a partir dos 35 anos $^{16}$. A amostra estudada evidenciou um predomínio dos potenciais doadores do sexo masculino com 156 casos $(78,79 \%)$ em relação ao sexo feminino, com $42(21,21 \%)$. Dos fatores de risco que predispõem ao trauma, o que se observa é a maior exposição dos homens a situações potencialmente traumáticas, sendo que no sexo masculino, o risco é duas a três vezes maior em comparação ao sexo feminino ${ }^{17}$. Estudo sobre mortalidade violenta no município de São Paulo encontrou predominância acentuada do sexo masculino: $90,2 \%$ do total de mortes por causas externas ${ }^{16}$. No mundo inteiro, traumas por acidente de trânsito são a principal causa de morte e o motivo principal de doenças em pessoas do sexo masculino ${ }^{18}$.

No presente estudo, a raça predominante entre as vítimas de trauma foi a raça branca com 133 casos $(67,17 \%)$ em relação à parda com 45 $(22,73 \%)$, negra com $19(9,60 \%)$ e a raça amarela com um $(0,50 \%)$. Segundo dados da Pesquisa Nacional por Amostras de Domicílios - PNAD, realizada pelo IBGE, no ano de 2004 a população do Estado de São Paulo era em sua grande maioria formada por brancos (70\%), seguida por pardos $(23 \%)$, negros $(5 \%)$ e índios $(0,5 \%)$; confirmando os achados desta pesquisa ${ }^{19}$.

O grande entrave no processo de disponibilização de órgãos e tecidos para transplante é representado pelo alto índice de não efetivação dos potenciais doadores $(72,73 \%)$ conforme representado no gráfico 3. A alta taxa de perda dos potenciais doadores deve-se em parte à própria estrutura do sistema de saúde brasileiro, que muitas vezes mantém potenciais doadores de órgãos e tecidos sob tratamento inadequado e ineficaz para a situação do doente. Enquanto num país como a Espanha o índice de doadores efetivos em morte encefálica é de 31,5 doadores por milhão de habitantes, no Brasil esse índice não chega a quatro doadores $/$ milhão $^{20}$. No estado de São Paulo o número é de 9,2 doadores por milhão de habitantes ${ }^{21}$.

Dentre os motivos de não-doação, o gráfico 4 mostra que a recusa familiar $(45,83 \%)$ e a parada cardíaca $(41,67 \%)$ representam o grande obstáculo na efetivação de potenciais doadores em nossa região (OPO-HC). Estudos mostram a recusa familiar como uma das principais causas de não efetivação da doação, e no Brasil, o índice ainda é considerado elevado, pois os padrões internacionais estabelecem como aceitáveis taxas em torno de $20 \%{ }^{8}$. Outro problema de não efetivação da doação é representado pelo alto índice de parada cardíaca. Isto ocorre devido a vários fatores, como por exemplo, demora na notificação do potencial doador, má manutenção do mesmo, entre outros. É preciso melhorar a notificação e a manutenção dos potenciais doadores, pois de cada dez potenciais doadores, somente um é notificado, o que faz com que a média do Brasil seja de apenas seis potenciais doadores por milhão de habitantes ${ }^{22}$.

No presente estudo, do total de 198 potenciais doadores, apenas 54 $(27,27 \%)$ foram doadores efetivos, sendo que dos órgãos captados para transplante, os rins foram os órgãos mais utilizados com $98,14 \%$, em comparação aos demais órgãos, como fígado (81,49\%), pâncreas $(51,85 \%)$, coração $(42,59 \%)$ e pulmões $(1,85 \%)$. Tal fato decorre de certas vantagens que os rins possuem para utilização comparada aos demais órgãos, tais como: não há limite de idade para utilização dos rins se o valor laboratorial da creatinina for normal, eles podem ser retirados de doadores sem batimentos cardíacos, e possuem um tempo de isquemia fria mais prolongado (o órgão fora do corpo sob refrigeração pode ser utilizado para transplante por até 36 horas) ${ }^{23}$. Dos tecidos, as córneas foram as que tiveram maior utilização com 74,07\% e os ossos a menor, com 1,85\%; o primeiro pela facilidade da retirada/utilização e aceitação da família; o último, possivelmente pela dificuldade de aceitação da família e descarte pelas equipes médicas (critérios rigorosos para utilização dos ossos). É importante destacar que a legislação brasileira requer a autorização por parte da família para doação de cada órgão e tecido.

\section{CONCLUSÃO}

O estudo sobre trauma e doação de órgãos e tecidos para transplante é de fundamental importância, em face do problema da crescente desproporção do número de pacientes em lista de espera versus número de transplantes realizados.

No Brasil, a doação de órgãos e tecidos ainda é um assunto polêmico. Dentro ou fora dos hospitais e até entre profissionais de saúde, há falta de informações sobre o processo de doação para transplante. Muitos profissionais desconhecem o efetivo e adequado processo de doação, sua sistematização e regulamentação ${ }^{24}$.

Nesta pesquisa em particular, foram estudados apenas pacientes vítimas de trauma que evoluíram com diagnóstico de morte encefálica, e que passaram pelo processo de doação-transplante. Entretanto, observam-se nos dados que a questão merece atenção do governo, dos transplantadores e dos pesquisadores, uma vez que grande parte dos potenciais doadores $(47,48 \%)$ era constituída de jovens na faixa etária entre 0 e 25 anos; a maioria (72,73\%) não foi doadora, e dos motivos da não-doação de órgãos e tecidos, a recusa familiar e a parada cardíaca atingiram taxas inaceitáveis comparadas com às do padrão internacional. Fica evidente que o foco de atenção deveria ser dirigido à notificação precoce desses potenciais doadores e à melhoria da manutenção dos mesmos, uma vez que a redução do índice de recusa familiar seria obtida em longo prazo, com programas voltados para a educação e conscientização da população sobre a questão da doação. 
O governo, em parceria com os meios de comunicação, deveria realizar campanhas permanentes que esclarecessem a importância da doação de órgãos e tecidos para transplante. Além disso, é necessário implementar uma política de educação continuada aos profissionais da saúde quanto ao processo de doação-transplante e de todos os desdobramentos decorrentes do desconhecimento desse processo. Com base no presente estudo, recomenda-se a realização de novos trabalhos que retratem a questão da recusa familiar, manutenção dos potenciais doadores, notificação precoce, bem como a educação voltada à população e aos profissionais da saúde.

\section{ABSTRACT}

Presently, trauma represents one of the major causes of death. Some victims with severe trauma may evolve to brain death, thus becoming potential organ donors. Purposes: To characterize different types of trauma which result in brain death; to characterize potential organ donors victims of trauma, according to their age, gender and race; to quantify the amount of potential organ donors victims of trauma who became actual donors, specifying the reasons for the non-donation, and to identify which organs and tissues have been used from trauma victim donors. Methods: A quantitative, descriptive and retrospective study using data collected from the record sheets of patients from the Organ Procurement Organization (OPO) of the Hospital das Clínicas of the Faculdade de Medicina da Universidade de São Paulo. Results: Data from 198 potential donors where assessed from January, 2004 through December, 2005. Data showed that from potential donors victims of trauma, $78.79 \%$ were male, $67.17 \%$ were Caucasian, and the predominant age varied between 0 to 25 years (47.48\%). Among different types of trauma, car accidents represented $44.45 \%$. From all potential donors, $72.73 \%$ did not accomplish the donation, and the main reason to the non-donation was family refusal (45.83\%), and cardiac arrest (41.67\%). Kidneys represented the majority of used organs $(98.14 \%)$. Conclusion: Our results showed that family refusal and cardiac arrest are undoubtedly the major reasons to the non-donation. It is clear that this theme deserves further attention both from the society, government and health care professionals.

Keywords: Transplantation, Injuries, Brain Death And Nursing.

\section{REFERENCES}

1. Coimbra RSM, Soldá SC, Casaroli AA, Rasslan S. Emergências traumáticas e não traumáticas: manual do residente e do estudante. São Paulo: Atheneu; 2001.

2. Knobel E. Condutas no paciente grave. São Paulo: Atheneu; 1998.

3. Souza HP. Cirurgia do trauma: condutas diagnósticas e terapêuticas. São Paulo: Atheneu; 2003.

4. Boletim final do $26^{\circ}$ Congresso Brasileiro de Cirurgia; 2006 fev 17; São Paulo. [texto na internet]. São Paulo: CBC; 2006. [citado 2007 ago 24]. Disponível em: http:// www.cbc.org.br.

5. Kotwica Z, Jakubowsky JR. Head-injured adult patients with Gcs of 3 on admission. Who have a chance to survive? Acta Neurochir (wien). 1995;133:56-9.

6. Conselho Federal de Medicina. Resolução CFM n ${ }^{\circ}$ 1.480, de 8 de agosto de 1997. Critérios de morte encefálica. Brasília, 8 ago. 1997.

7. Brasil. Senado Federal. Subsecretaria de Informações. Lei ${ }^{\circ} 9.434$, de 4 de fevereiro de 1997. Dispõe sobre a remoção de órgão, tecidos e partes do corpo humano para fins de transplante e tratamento, e da outras providências. Diário Oficial da União, Brasília, 5 fev. 1997. Seção 1.

8. Garcia VD. Por uma política de transplante no Brasil. São Paulo: Office; 2000.

9. Brasil. Senado Federal. Subsecretaria de Informações. Lei no 10.211, de 23 de março de 2001. Altera dispositivos da Lei n. 9.434, de 4 de fevereiro de 1997, que dispõe sobre a remoção de órgãos, tecidos e partes do corpo humano para fins de transplante e tratamento. Diário Oficial da União, Brasília, 24 mar. 2001. Seção 1-E, p 6.

10. Rampazzo L. Metodologia da pesquisa. São Paulo: Loyola; 2002.

11. Contandriopoulos AP. Saber preparar uma pesquisa. São Paulo, Rio de Janeiro: Hucitec Abrasco; 1994.
12. Severino AJ. Metodologia do trabalho científico. São Paulo: Cortez; 2002.

13. Macedo AC, Paim JS, Silva LMV, Costa MCN. Violência e desigualdade social: mortalidade por homicídio e condições de vida em Salvador, Brasil. Rev Saúde Pública. 2001;35(6):515-22.

14. Advanced Trauma Life Support (ATLS). Student manual. Chicado: American College of Surgeon; 2004.

15. Rede Globo - SPTV. Vítimas do trânsito. [vídeo na internet]. São Paulo: Rede Globo; 2007. [Citado 2007 ago 24]. Disponível em: http//sptv.globo.com/sptv

16. Gawryszewski VP, Jorge MHPM. Mortalidade violenta no Município de São Paulo nos últimos 40 anos. Rev Bras Epidemiol. 2000;3(1):50-69.

17. Pereira CU. Neurotraumatologia. Rio de Janeiro: Revinter; 2000.

18. Atendimento pré-hospitalar ao traumatizado: básico e avançado. Prehospital trauma life support (PHTLS). $5^{\mathrm{a}}$ ed. Rio de Janeiro: Elsevier; 2004.

19. Instituto Brasileiro de Geografia e Estatística.(IBGE). População e domicílio: PAND 2004. [texto na internet]. [Citado 2007 ago 24]. Disponível em: http//www.ibge.gov. br/estadosat/temas.

20. Segre M. A questão ética e a saúde humana. São Paulo: Atheneu; 2006.

21. Associação Brasileira de Transplante de Órgãos (ABTO). Reg Bras Transplant. (RBT). 2005;11(2).

22. Associação Brasileira de Transplantes de Órgãos (ABTO). Bol Informat. 2005;1:14-15.

23. Associação Brasileira de Transplante de órgãos (ABTO). I Reunião de diretrizes básicas para captação e retirada de múltiplos órgãos e tecidos. São Paulo: Ipsis; 2003.

24. Lima AAF, Moraes EL, Padrão MB. Fatores que influenciam a recusa familiar no processo de doação de órgãos e tecidos para transplantes. J Bras Transpl. 2004;7(3):137-140. 